\title{
CONVERGENCE ANALYSIS OF GENERALIZED ITERATIVELY REWEIGHTED LEAST SQUARES ALGORITHMS ON CONVEX FUNCTION SPACES*
}

\author{
NICOLAI BISSANTZ ${ }^{\dagger}$, LUTZ DÜMBGEN ${ }^{\ddagger}$, AXEL MUNK ${ }^{\S}$, AND BERND STRATMANN ${ }^{\dagger}$
}

Yehuda Vardi passed away unexpectedly on Jan. 13th, 2005, and we would like to dedicate this work to his memory.

\begin{abstract}
The computation of robust regression estimates often relies on minimization of a convex functional on a convex set. In this paper we discuss a general technique for a large class of convex functionals to compute the minimizers iteratively, which is closely related to majorizationminimization algorithms. Our approach is based on a quadratic approximation of the functional to be minimized and includes the iteratively reweighted least squares algorithm as a special case. We prove convergence on convex function spaces for general coercive and convex functionals $F$ and derive geometric convergence in certain unconstrained settings. The algorithm is applied to total variation (TV) penalized quantile regression and is compared with a step size corrected NewtonRaphson algorithm. It is found that typically in the first steps the iteratively reweighted least squares algorithm performs significantly better, whereas the Newton type method outpaces the former only after many iterations. Finally, in the setting of bivariate regression with unimodality constraints we illustrate how this algorithm allows one to utilize highly efficient algorithms for special quadratic programs in more complex settings.
\end{abstract}

Key words. regression analysis, monotone regression, quantile regression, shape constraints, $L^{1}$ regression, nonparametric regression, total variation semi-norm, reweighted least squares, Fermat's problem, convex approximation, quadratic approximation, pool adjacent violators algorithm

AMS subject classifications. 62G07, 62J05, 65K05, 85-08

DOI. $10.1137 / 050639132$

1. Introduction. The computation of robust parametric and nonparametric regression estimators often requires the minimization of (convex) functionals on a set $\mathcal{C}$ which is determined by a priori information on the model underlying the data. For example, $\mathcal{C}$ can be a linear finite-dimensional space (linear model) or the set of isotonic vectors $m=\left(m_{1}, \ldots, m_{d}\right) \in \mathbb{R}^{d}, m_{1} \leq \ldots \leq m_{d}$, with $d \leq n$. To this end the functional

$$
F^{(\rho)}(m)=\sum_{i=1}^{n} \rho\left(r_{i}(m)\right)
$$

has to be minimized over $\mathcal{C} \subset \mathbb{R}^{d}$. Here $r_{1}, \ldots, r_{n}$ denote the (model-dependent) residuals of $n$ data pairs $\left(X_{i}, Y_{i}\right), 1 \leq i \leq n$, and $\rho$ a given loss function [17]. Taking

\footnotetext{
* Received by the editors August 29, 2005; accepted for publication (in revised form) November 4, 2008; published electronically February 20, 2009. Financial support of the Swiss National Science Foundation, of the DFG by the grants Graduiertenkolleg 1023, SFB 475 and FOR 916, and by the DAAD is gratefully acknowledged.

http://www.siam.org/journals/siopt/19-4/63913.html

${ }^{\dagger}$ Fakultät für Mathematik, Universitätsstraße 150, Mathematik III, NA 3/70, D-44780 Bochum, Germany (nicolai.bissantz@rub.de).

${ }^{\ddagger}$ Institute of Mathematical Statistics and Actuarial Science, University of Bern, 3012 Bern, Switzerland (dumbgen@stat.unibe.ch).

$\S$ Institute for Mathematical Stochastics, Georg-August-University Göttingen, 37073 Göttingen, Germany (munk@math.uni.goettingen.de, bstrat@gmx.net).
}

1828 
$\rho(z)=z^{2} / 2$ gives the ordinary least squares problem, while

$$
\rho(z)=2|z| \cdot\left\{\begin{array}{cc}
p & z \geq 0 \\
1-p & z<0
\end{array}\right.
$$

with $0<p<1$ yields quantile regression [21,30]. Other functions are Huber's [16] loss function

$$
\rho(z)=\left\{\begin{array}{cc}
z^{2} / 2 & |z| \leq \gamma \\
\gamma|z|-\gamma^{2} / 2 & |z|>\gamma
\end{array}\right.
$$

or the logistic loss function $\rho(z)=\gamma^{z} \log (\cosh (z / \gamma))$ [7] for some $\gamma>0$. An important extension of (1) are functionals

$$
F(m)=F^{(\rho)}(m)+\lambda P(m), \quad \lambda \geq 0,
$$

where $P(m)$ denotes a penalizing term such as, for instance, the discrete total variation semi-norm of $m \in \mathbb{R}^{d}$,

$$
P(m)=\sum_{j=1}^{d-1}\left|m_{j}-m_{j+1}\right| ;
$$

see $[23,22]$ or $[27]$. In this paper a generalization of the iteratively reweighted least squares (IRLS) algorithm - therefore named GIRLS - is considered for minimization of a functional $F$ as in (3) over any convex subset $\mathcal{C}$ of $\mathbb{R}^{d}$. This allows us to extend the IRLS algorithm, for example, to situations where $\mathcal{C}$ is defined as the space of monotone (or $k$-modal) vectors or to the problem of nonparametric regression estimates with total variation semi-norm penalization of its discrete derivative.

The general idea of the IRLS algorithm (and variants of it) is to approximate the functional $F$ in a first step by smooth functionals $F_{\delta}$ such that $F_{\delta} \rightarrow F$ pointwise as $\delta \searrow 0$. The collection $\left(F_{\delta}\right)_{\delta>0}$ will be called a regularization of $F$ (cf. Def. 1). In a second step, for each given base point $f \in \mathcal{C}$ the functional $F_{\delta}$ will be approximated by $G_{\delta}(f,$.$) (cf. Def. 2). Here G_{\delta}: \mathcal{C} \times \mathbb{R}^{d} \rightarrow \mathbb{R}$ is a functional which is chosen such that a quick and numerically stable minimization can be performed. The resulting minimizer will serve as an approximation for the minimizer $m_{\delta}^{*}$ of $F_{\delta}$ and hence for a minimizer $m^{*}$ of $F$. In particular, if it is possible to choose $G_{\delta}$ as a polynomial of degree two, the well-known IRLS algorithm may result [25, 28, 10].

The GIRLS algorithm can be summarized schematically as follows:

Preliminary SteP: Determine a regularization $\left(F_{\delta}\right)_{\delta>0}$ of $F$ and corresponding smooth approximations $G_{\delta}, \delta>0$.

STEP 1: Initialize $\delta>0$ and $m_{\delta}^{(0)} \in \mathcal{C}$.

STEP 2: Repeat the following procedure until $\delta$ is sufficiently small: Compute

$$
m_{\delta}^{(k)}:=\underset{m \in \mathcal{C}}{\operatorname{argmin}} G_{\delta}\left(m_{\delta}^{(k-1)}, m\right) \quad \text { for } k=1,2,3, \ldots
$$

and terminate this iteration for a proper $k=k(\delta)$. Then replace $\left(\delta, m_{\delta}^{(0)}\right)$ with $\left(\delta / 2, m_{\delta}^{(k(\delta))}\right)$.

Output: The final $m_{\delta}^{(k(\delta))}$ is our approximate minimizer of $F$ over $\mathcal{C}$.

A more detailed description of this algorithm, including pseudocode and an explicit rule for $k(\delta)$ is provided in section 3.2.

The IRLS and related algorithms are based on the idea of majorizing functionals by a sequence of quadratic approximations and subsequent minimization. These have been treated extensively in the literature, e.g., [24, 19, 42, 29, 9, 18, 36, 37], and the 
references therein. However, in most cases convergence is only shown for $\mathcal{C}=\mathbb{R}^{d}$. This simplifies proofs notably, since the minimizers can be represented as zeros of the derivatives of the functional. For arbitrary convex $\mathcal{C}$, however, the minimizers are no longer represented solely by such equality constraints, instead inequalities occur. Notable exceptions for general convex $\mathcal{C}$ are in [13], where however, the convergence results are restricted to a special class of functionals, requiring, e.g., $F(m)=O(\|m\|)$, or [39], who show convergence on convex polyhedral sets under the assumption that $F$ is two times differentiable. Our findings generalize these results to the case of $\mathcal{C}$ being an arbitrary convex closed set as well as to more general functionals which are only required to be coercive and convex. This appears to be close to the weakest possible set of assumptions required for a general proof of convergence. Our proof adopts various arguments from convex analysis.

It is interesting to note that in the numerical literature the IRLS algorithm is denoted as the Weiszfeld algorithm [40, 41] who suggested this algorithm to solve the Fermat-Steiner-Weber problem [40, 41, 24, 19], which is known to the statistical community as the computation of the spatial median (as mentioned in $[5,6,11]$ ).

The remainder of this paper is organized as follows. First, we motivate the GIRLS algorithm for the special case of $L^{1}$-regression in section 2 . Then we present the GIRLS algorithm in a general framework and prove various results about its convergence in section 3. Its convergence to the minimizer $m_{\delta}^{*}$ and hence to $m^{*}$ as $\delta \searrow 0$ will be shown under very general assumptions (Theorem 2). Furthermore, in Theorem 5 we prove geometric, or, more precisely, at least $Q$-linear convergence of the sequence $\left(m_{k}\right)_{k}$ to $m_{\delta}^{*}$ under slightly stronger conditions (cf. [39] and [3]), and guidance is provided on the choice of the number of iterates in (5) and the regularization parameter $\delta$. Finally, we show in Theorem 3 that any convex and coercive functional $F$ can be regularized by a sequence $F_{\delta}$ s.t. each $F_{\delta}$ admits a quadratic approximation $G_{\delta}$ from above.

We stress that an advantage of the GIRLS approach is flexibility in the choice of $F_{\delta}$ and $G_{\delta}$. This choice can be driven by various aspects, such as computational efficiency or rate of convergence (cf. Theorem 3). In this paper we emphasize the possibility to make use of efficient algorithms already available for the minimization of $G_{\delta}$, such as the pool adjacent violators algorithm (PAVA) for isotonic weighted least squares approximation (see [33] for a comprehensive treatment). This is illustrated in section 4, where we describe the construction of $F_{\delta}$ and $G_{\delta}$ in some specific cases explicitly. In section 5 we discuss two numerical examples. In the first example we investigate in detail numerical performance of the GIRLS algorithm for the case of total variation (TV) penalized quantile regression. To this end the GIRLS algorithm is compared with a step size corrected Newton-Raphson algorithm. It is found that typically it outperforms the latter one in the first iteration steps significantly, in particular when the initial value is far from the optimum. This finding coincides with other numerical experiments, e.g., when applying the algorithms to $L^{1}$-penalized Poisson regression.

In the second example we apply the GIRLS algorithm to a two-dimensional TV minimization where we impose an additional unimodality constraint in one direction. We show that the GIRLS algorithm allows us to include the PAVA for the univariate unimodal subproblem, which is in general not possible for regression with two- or higher dimensional predictor. Note also that PAVA type methods are not available in general if an additional penalization term as in (3) is added. Again, the GIRLS algorithm offers a possibility to include them in each updating step.

In summary, the main advantage of the GIRLS algorithm is twofold. First, it is simple to perform and offers great flexibility for the choice of the approximating functionals $G_{\delta}$. Second, it allows us to combine various restrictions and minimization 
criteria (such as monotonicity constraints and roughness penalties). For such complex minimization problems, simple and quick algorithms such as PAVA or Newton type algorithms are not available in general, and more complicated and time consuming algorithms such as quadratic programming or interior point methods become necessary. Here the GIRLS algorithm represents a feasible alternative because it typically requires in each updating step the computation of minimizers (e.g., a weighted $L^{2}$ solution), which can be obtained easily. Further, our numerical experiments have shown that a rather small number of updating steps give already satisfactory results and the GIRLS algorithm outperfoms competitors in the first iterations, which is in accordance with previous numerical findings (see, e.g., [39]). Hence, as a practical rule of thumb, we find that the GIRLS algorithm is very simple to implement and provides a quick improvement of an initial value by a few iterations. It can be improved additionally by performing subsequent iterations by other, more sophisticated, optimization algorithms.

2. $\boldsymbol{L}^{\mathbf{1}}$-regression with the GIRLS algorithm. As a motivating example consider the $L^{1}$ linear regression problem for observations $\left(X_{1}, Y_{1}\right),\left(X_{2}, Y_{2}\right), \ldots,\left(X_{n}, Y_{n}\right)$ in $\mathbb{R}^{d} \times \mathbb{R}$. Assuming that $Y_{i}$ equals $X_{i}^{\top} m$ plus a random error, the goal is to compute

$$
m:=\underset{m \in \mathbb{R}^{d}}{\operatorname{argmin}} \sum_{i=1}^{n}\left|Y_{i}-X_{i}^{\top} m\right|=\underset{m \in \mathbb{R}^{d}}{\operatorname{argmin}} F(m),
$$

an estimator of the unknown parameter vector $m \in \mathbb{R}^{d}$. Iteratively reweighted least squares is based on the idea that, in a first step, the $L^{1}$-norm $F$, being a convex functional, will be approximated (regularized) by a family of smooth convex functionals $F_{\delta}, \delta>0$, e.g.,

$$
F_{\delta}(m)=\sum_{i=1}^{n} h_{\delta}\left(Y_{i}-X_{i}^{\top} m\right)
$$

where

$$
h_{\delta}(z)=\left[z^{2}+\delta\right]^{1 / 2} .
$$

The regularization of a nonsmooth functional as in (6) by (7) is well known, of course (see, e.g., [38]). It is supposed that minimization of $F_{\delta}$ is numerically better tractable than minimization of the original functional $F$ in in (6). Then $m_{\delta}:=$ $\operatorname{argmin}_{m \in \mathbb{R}^{d}} F_{\delta}(m)$ will be an approximation of $m$ (cf. Theorem 1). In order to compute $m_{\delta}$ the following recursion formula is iterated:

$$
m_{\delta}^{(k+1)}=\underset{m \in \mathbb{R}^{d}}{\operatorname{argmin}} \sum_{i=1}^{n} \frac{\left(Y_{i}-X_{i}^{\top} m\right)^{2}}{h_{\delta}\left(Y_{i}-X_{i}^{\top} m_{\delta}^{(k)}\right)} .
$$

Note that in each updating step the computation of $m_{\delta}^{(k+1)}$ means solving a simple diagonally reweighted least squares minimization problem, which can easily be done by using standard methods such as, e.g., Householder $Q R$ decomposition. As a starting value $m_{\delta}^{(0)}$ any (reasonable) choice, e.g., the least squares estimator, may serve.

It is instructive to indicate a proof for this simple case. The basic idea is to approximate $h_{\delta}(z)$ from above for any given real number $r$ by a quadratic function $g_{\delta}(r, z)=c(r)+a(r) z^{2} / 2$ of $z$ such that $g_{\delta}(r, \cdot) \geq h_{\delta}$ and $g_{\delta}(r, r)=h_{\delta}(r)$. This can 
be achieved indeed with

$$
g_{\delta}(r, z)=h_{\delta}(r)+h_{\delta}(r)^{-1}\left(z^{2}-r^{2}\right) / 2 ;
$$

see also Lemma 1 in section 4 . The intrinsic reason is that $h_{\delta}$ is an even convex function whose second derivative $h_{\delta}^{\prime \prime}$ is nonincreasing on $[0, \infty)$. Thus $m_{\delta}^{(k+1)}$ in $(8)$ is the minimizer of

$$
G_{\delta}\left(m_{\delta}^{(k)}, m\right):=\sum_{i=1}^{n} g_{\delta}\left(Y_{i}-X_{i}^{\top} m_{\delta}^{(k)}, Y_{i}-X_{i}^{\top} m\right)
$$

over all $m \in \mathbb{R}^{d}$. Note that $F_{\delta}$ as well as $G_{\delta}\left(m_{\delta}^{(k)}, \cdot\right)$ are convex functions such that $F_{\delta}(m) \leq G_{\delta}\left(m_{\delta}^{(k)}, m\right)$ with equality for $m=m_{\delta}^{(k)}$, and their gradients satisfy

$$
\nabla F_{\delta}\left(m_{\delta}^{(k)}\right)=\nabla G_{\delta}\left(m_{\delta}^{(k)}, m_{\delta}^{(k)}\right) .
$$

Here and in the following the gradient of $G_{\delta}$ is defined with respect to the second argument. Thus

$$
F_{\delta}\left(m_{\delta}^{(k+1)}\right) \leq G_{\delta}\left(m_{\delta}^{(k)}, m_{\delta}^{(k+1)}\right) \leq G_{\delta}\left(m_{\delta}^{(k)}, m_{\delta}^{(k)}\right)=F_{\delta}\left(m_{\delta}^{(k)}\right),
$$

and the second inequality in the latter display is strict if, and only if, $m_{\delta}^{(k)}$ differs from the solution $m_{\delta}$. Consequently, $F_{\delta}\left(m_{\delta}^{(k)}\right)$ is either strictly decreasing in $k$, or $m_{\delta}^{(k)}=m_{\delta}$ for sufficiently large $k$. This fact was established by [25] for the particular problem (6). Convergence of $m_{\delta}^{(k)}$ to $m_{\delta}$ as $k \rightarrow \infty$ follows from our general Theorem 2 below.

\section{The GIRLS algorithm.}

3.1. Main theorem and convergence analysis. Returning to the general setting, we always assume that our target functional $F: \mathbb{R}^{d} \rightarrow \mathbb{R}$ is convex and coercive, i.e., $F(x) \rightarrow \infty$ as $\|x\| \rightarrow \infty$. Moreover, let $\mathcal{C} \subset \mathbb{R}^{d}$ be closed and convex. This entails that the set

$$
M^{*}:=\underset{m \in \mathcal{C}}{\operatorname{argmin}} F(m)
$$

is a nonvoid, compact, and convex subset of $\mathcal{C}$. Now the first step is to approximate $F$ by a family of strictly convex and smooth functionals $F_{\delta}, \delta>0$, converging pointwise to $F$ as $\delta \searrow 0$. This is summarized in the following definition.

Definition 1. A functional $F_{\delta}: \mathbb{R}^{d} \rightarrow \mathbb{R}$ is called regular, if $F_{\delta}$ is strictly convex, continuously differentiable, and coercive. A regularization of $F$ consists of regular functionals $F_{\delta}, \delta>0$, such that $F_{\delta}$ converges pointwise to $F$ as $\delta \searrow 0$.

Theorem 4 below shows that there exists always a regularization $\left(F_{\delta}\right)_{\delta>0}$ for $F$. It follows from strict convexity and coercivity of $F_{\delta}$ that it has a unique minimizer

$$
m_{\delta}^{*}:=\underset{m \in \mathcal{C}}{\operatorname{argmin}} F_{\delta}(m)
$$

which serves as an approximation to $M^{*}$. The next theorem provides an exact formulation of this fact. 
TheOREM 1 (approximation of $M^{*}$ ). Let $F: \mathbb{R}^{d} \rightarrow \mathbb{R}$ be a convex and coercive functional, and let $\left(F_{\delta}\right)_{\delta>0}$ be a regularization of $F$. Then, as $\delta \searrow 0$,

$$
\left.\begin{array}{c}
F_{\delta}\left(m_{\delta}^{*}\right) \\
F\left(m_{\delta}^{*}\right)
\end{array}\right\} \rightarrow \min _{x \in \mathcal{C}} F(x) \quad \text { and } \quad d\left(m_{\delta}^{*}, M^{*}\right):=\inf _{y \in M^{*}}\left\|m_{\delta}^{*}-y\right\| \rightarrow 0 .
$$

Before proving Theorem 1 we summarize some well-known facts about convex functionals (see [34]), which we utilize in the subsequent proofs. A convex functional on $\mathbb{R}^{d}$ is automatically continuous. If a sequence of convex functionals on $\mathbb{R}^{d}$ converges pointwise, then the convergence is uniform on arbitrary bounded sets. Finally, if $H: \mathbb{R}^{d} \rightarrow \mathbb{R}$ is convex and differentiable, and if $\mathcal{C} \subset \mathbb{R}^{d}$ is closed and convex, then $f \in \mathcal{C}$ minimizes $H$ over $\mathcal{C}$ if, and only if,

$$
\nabla H(f)^{\top}(m-f) \geq 0 \quad \text { for all } m \in \mathcal{C} .
$$

Proof of Theorem 1. For any set $S \subset \mathbb{R}^{d}$ let $\left\|F-F_{\delta}\right\|_{S}$ be the supremum norm of $F-F_{\delta}$ over $S$. Since $M^{*}$ is compact, for any fixed $\epsilon>0$, the set $B_{\epsilon}:=\{m \in$ $\left.\mathcal{C}: d\left(m, M^{*}\right) \leq \epsilon\right\}$ is compact, too. Thus $\left\|F-F_{\delta}\right\|_{B_{\epsilon}}$ tends to zero as $\delta \searrow 0$. In particular, for sufficiently small $\delta>0$,

$$
\min _{m \in \mathcal{C}: d\left(m, M^{*}\right)=\epsilon} F_{\delta}(m)>\max _{m \in M^{*}} F_{\delta}(m) .
$$

To verify (11), first note that it holds with $F$ in place of $F_{\delta}$, by definition of $M^{*}$. Since $F_{\delta} \rightarrow F$ uniformly on $B_{\epsilon}$, (11) holds for sufficiently small $\delta>0$. But (11) implies that $F_{\delta}\left(m_{o}\right)>\min _{m \in M^{*}} F_{\delta}(m)$ for any $m_{o} \in \mathcal{C} \backslash B_{\epsilon}$, and hence $m_{\delta}^{*} \in B_{\epsilon}$. Let $m_{*}$ be the metric projection of $m_{o}$ onto $M^{*}$ and write $m_{o}=m_{*}+t v$ for some unit vector $v \in \mathbb{R}^{d}$ and a scalar $t>\epsilon$. Then it follows from convexity of the function $t \mapsto F_{\delta}\left(m_{*}+t v\right)$ that

$$
\begin{aligned}
F_{\delta}\left(m_{o}\right)-\min _{m \in M^{*}} F_{\delta}(m) & \geq F_{\delta}\left(m_{*}+t v\right)-F_{\delta}\left(m_{*}\right) \\
& \geq(t / \epsilon)\left(F_{\delta}\left(m_{*}+\epsilon v\right)-F_{\delta}\left(m_{*}\right)\right) \\
& >0
\end{aligned}
$$

in case of (11). These considerations show already that $d\left(m_{\delta}^{*}, M^{*}\right) \rightarrow 0$ as $\delta \searrow 0$. Note also that in case of $m_{\delta}^{*} \in B_{\epsilon}$,

$$
\left|F_{\delta}\left(m_{\delta}^{*}\right)-F\left(m_{\delta}^{*}\right)\right| \leq\left\|F-F_{\delta}\right\|_{B_{\epsilon}},
$$

and

$$
F\left(m_{\delta}^{*}\right)-\min _{x \in \mathcal{C}} F(x) \leq \max _{x, y \in B_{\epsilon}:\|x-y\| \leq \epsilon}|F(y)-F(x)| .
$$

Finally, the r.h.s. of the latter inequality tends to 0 as $\epsilon \searrow 0$, by compactness of $M^{*}$ and continuity of $F$. These findings show that both $F\left(m_{\delta}^{*}\right)$ and $F_{\delta}\left(m_{\delta}^{*}\right)$ tend to $\min _{x \in \mathcal{C}} F(x)$ as $\delta \searrow 0$.

The second step is to determine $m_{\delta}^{*}$ via approximations $G_{\delta}(f, \cdot)$ of $F_{\delta}$ for various $f \in \mathcal{C}$ as in (5). The following definition summarizes our assumptions on $G_{\delta}$.

Definition 2. Let $F_{\delta}: \mathbb{R}^{d} \rightarrow \mathbb{R}$ be a regular functional. Another functional $G_{\delta}: \mathcal{C} \times \mathbb{R}^{d} \rightarrow \mathbb{R}$ is called a smooth approximation of $F_{\delta}$ from above, if it is continuous in both arguments and satisfies the following additional properties for arbitrary $f \in \mathcal{C}$ :

Copyright $@$ by SIAM. Unauthorized reproduction of this article is prohibited. 
(i) $G_{\delta}(f, \cdot)$ is strictly convex and continuously differentiable,

(ii) $G_{\delta}(f, m) \geq F_{\delta}(m)$ for all $m \in \mathbb{R}^{d}$ with equality for $m=f$.

The functional $G_{\delta}$ is called a quadratic approximation of $F_{\delta}$ from above if, in addition, $G_{\delta}(f, \cdot)$ is always a polynomial of order two, i.e.,

$$
G_{\delta}(f, m)=F_{\delta}(f)+\nabla F_{\delta}(f)^{\top}(m-f)+2^{-1}(m-f)^{\top} B(f)(m-f)
$$

for some symmetric, positive definite matrix $B(f) \in \mathbb{R}^{d \times d}$.

The next theorem is the main result of this paper.

ThEOREM 2 (convergence of the GIRLS algorithm). Let $\mathcal{C} \subset \mathbb{R}^{d}$ be a closed convex set and $F_{\delta}: \mathbb{R}^{d} \rightarrow \mathbb{R}$ be a regular functional which can be smoothly approximated from above by $G_{\delta}$. Then the GIRLS algorithm, defined by (5) with an arbitrary starting point $m_{\delta}^{(0)} \in \mathcal{C}$, yields a sequence $\left(m_{\delta}^{(k)}\right)_{k=0}^{\infty}$ converging to $m_{\delta}^{*}$.

Proof. At first we prove that $F_{\delta}\left(m_{\delta}^{(k)}\right)$ is decreasing in $k$. It follows from Property (ii) in Definition 2 that the gradients $\nabla F_{\delta}(m)$ and $\nabla G_{\delta}\left(m_{\delta}^{(k)}, m\right)$ (defined with respect to the second argument) coincide for $m=m_{\delta}^{(k)}$. Thus it follows from the characterization (10), applied to $H=G_{\delta}\left(m_{\delta}^{(k)}, \cdot\right)$ and $H=F_{\delta}$, respectively, that $m_{\delta}^{(k+1)}=m_{\delta}^{(k)}$ if, and only if, $m_{\delta}^{(k)}=m_{\delta}^{*}$. Otherwise the minimizer $m_{\delta}^{(k+1)}$ of $G_{\delta}\left(m_{\delta}^{(k)}, \cdot\right)$ over $\mathcal{C}$ differs from $m_{\delta}^{(k)}$, whence Property (ii) in Definition 2 entails

$$
F_{\delta}\left(m_{\delta}^{(k+1)}\right) \leq G_{\delta}\left(m_{\delta}^{(k)}, m_{\delta}^{(k+1)}\right)<G_{\delta}\left(m_{\delta}^{(k)}, m_{\delta}^{(k)}\right)=F_{\delta}\left(m_{\delta}^{(k)}\right) .
$$

By monotonicity of $\left(F_{\delta}\left(m_{\delta}^{(k)}\right)\right)_{k}$, all points $m_{\delta}^{(k)}$ lie in the set $\left\{m \in \mathcal{C}: F_{\delta}(m) \leq\right.$ $\left.F_{\delta}\left(m_{\delta}^{(0)}\right)\right\}$, which is compact by continuity and coercivity of $F_{\delta}$. Hence it is sufficient to show that any limit point $m_{o}$ equals $m_{\delta}^{*}$. Now, take an arbitrary convergent subsequence $\left(m_{\delta}^{\left(k_{\ell}\right)}\right)_{\ell}$ with limit $m_{o}$. For any $v \in \mathcal{C}$,

$$
\begin{aligned}
F_{\delta}\left(m_{\delta}^{\left(k_{\ell}+1\right)}\right) & \leq G_{\delta}\left(m_{\delta}^{\left(k_{\ell}\right)}, m_{\delta}^{\left(k_{\ell}+1\right)}\right) \\
& \leq G_{\delta}\left(m_{\delta}^{\left(k_{\ell}\right)}, v\right) \\
& \rightarrow G_{\delta}\left(m_{o}, v\right) \quad \text { as } \ell \rightarrow \infty
\end{aligned}
$$

by continuity of $G_{\delta}$. But

$$
\lim _{\ell \rightarrow \infty} F_{\delta}\left(m_{\delta}^{\left(k_{\ell}+1\right)}\right) \geq \lim _{\ell \rightarrow \infty} F_{\delta}\left(m_{\delta}^{\left(k_{\ell+1}\right)}\right)=F_{\delta}\left(m_{o}\right)=G_{\delta}\left(m_{o}, m_{o}\right) .
$$

Thus $G_{\delta}\left(m_{o}, m_{o}\right) \leq G_{\delta}\left(m_{o}, v\right)$ for all $v \in \mathcal{C}$; i.e., $m_{o}$ is the unique minimizer of $G_{\delta}\left(m_{o}, \cdot\right)$. As argued above, this entails that $m_{o}=m_{\delta}^{*}$.

The next theorem states that convex and coercive functionals $F$ can always be regularized and approximated quadratically from above. Hence GIRLS is, in principle, always applicable.

ThEOREM 3 (regularization and approximation of $F$ ). Let $F: \mathbb{R}^{d} \rightarrow \mathbb{R}$ be a convex and coercive functional. Then there exists a regularization $\left(F_{\delta}\right)_{\delta>0}$ of $F$ such that each $F_{\delta}$ admits a quadratic approximation $G_{\delta}$ from above.

In order to prove Theorem 3, we require the following result.

THEOREM 4. Let $F$ be a nonnegative, coercive, convex functional on $\mathbb{R}^{d}$. Then there are strictly convex and infinitely often differentiable functionals $F_{\delta} \geq F, \delta>0$, such that $F_{\delta} \rightarrow F$ pointwise as $\delta \searrow 0$. 
Proof. Let $K(x):=1\{\|x\|<1\} C \exp \left(-\left(1-\|x\|^{2}\right)^{-1}\right)$, where $C$ is chosen such that $K$ integrates to one. This is a well-known example of an infinitely differentiable, nonnegative, even kernel function with compact support $\{x:\|x\| \leq 1\}$. For $\delta>0$ we define $K_{\delta}(x):=\delta^{-1} K\left(\delta^{-1} x\right)$ and

$$
F_{\delta}(x):=\int F(y) K_{\delta}(x-y) \mathrm{d} y=\int F(x+\delta z) K(z) \mathrm{d} z .
$$

It is well known that $F_{\delta}$ is infinitely often differentiable with limit $F$ pointwise (cf. [35]. It is also inherits convexity from $F$, because for $x, y \in \mathbb{R}^{d}$ and $\lambda \in(0,1)$,

$$
\begin{aligned}
F_{\delta}((1-\lambda) x+\lambda y) & =\int F_{\delta}((1-\lambda)(x+\delta z)+\lambda(y+\delta z)) K(z) \mathrm{d} z \\
& \leq \int((1-\lambda) F(x+\delta z)+\lambda F(y+\delta z)) K(z) \mathrm{d} z \\
& =(1-\lambda) F_{\delta}(x)+\lambda F_{\delta}(y) .
\end{aligned}
$$

Moreover, since $K$ is even,

$$
F_{\delta}(x)=\int \frac{F(x+\delta z)+F(x-\delta z)}{2} K(z) \mathrm{d} z \geq \int F(x) K(z) \mathrm{d} z=F(x),
$$

again by convexity of $F$. Finally, if $F_{\delta}$ fails to be strictly convex, we may add to $F_{\delta}$ the strictly convex function $x \mapsto \delta\|x\|^{2}$.

We mention that the construction of $F_{\delta}$ given here is mainly for theoretical purposes, and may in practice be difficult to evaluate numerically due to the high dimensionality of the integral.

Proof of Theorem 3. Let $\left(F_{\delta}\right)_{\delta>0}$ be a regularization of $F$ such that $D^{2} F_{\delta}$ is positive definite everywhere; cf. Theorem 4 and its proof. It may happen that $\limsup _{\|m\| \rightarrow \infty} F_{\delta}(m) /\|m\|^{2}=\infty$, rendering quadratic approximation of $F_{\delta}$ from above impossible. Thus we modify the functions $F_{\delta}$ as follows: Let

$$
c_{\delta}:=\max _{\|m\| \leq \delta^{-1}} \lambda_{\max }\left(D^{2} F_{\delta}(m)\right)
$$

with $\lambda_{\max }(A)$ denoting the largest eigenvalue of a symmetric matrix $A \in \mathbb{R}^{d \times d}$. Starting from the representation

$$
F_{\delta}(m)=F_{\delta}(0)+\nabla F_{\delta}(0)^{\top} m+\int_{0}^{1} m^{\top} D^{2} F_{\delta}(t m) m(1-t) d t,
$$

we define

$$
\tilde{F}_{\delta}(m):=F_{\delta}(0)+\nabla F_{\delta}(0)^{\top} m+\int_{0}^{1} m^{\top} \min \left(D^{2} F_{\delta}(t m), c_{\delta} I\right) m(1-t) d t .
$$

Here $\min \left(A, c_{\delta} I\right) \in \mathbb{R}^{d \times d}$ is obtained from the spectral representation of $A$ by replacing each eigenvalue $\lambda_{i}(A)$ with $\min \left(\lambda_{i}(A), c_{\delta}\right)$. Note that $\tilde{F}_{\delta}$ is twice continuously differentiable with $\tilde{F}_{\delta}(0)=F_{\delta}(0), \nabla \tilde{F}_{\delta}(0)=\nabla F_{\delta}(0)$, and $D^{2} \tilde{F}_{\delta}=\min \left(D^{2} F_{\delta}, c_{\delta} I\right)$. The Hessian matrix is positive definite with largest eigenvalue never exceeding $c_{\delta}$. In addition, $\tilde{F}_{\delta}=F_{\delta}$ on $\left\{m:\|m\| \leq \delta^{-1}\right\}$. Thus for sufficiently small $\delta>0, \tilde{F}_{\delta}$ is regular, and a quadratic approximation of $\tilde{F}_{\delta}$ from above is given by

$$
G_{\delta}(f, m):=\tilde{F}_{\delta}(f)+\nabla \tilde{F}_{\delta}(f)^{\top}(m-f)+c_{\delta}\|m-f\|^{2} / 2 .
$$


Remark 1. In Definition 1 we assume that $F_{\delta}$ is strictly convex. This property is only required for notational convenience, because it guarantees uniqueness of the minimizer $m_{\delta}^{*}$. A careful inspection of the proof of Theorem 1 shows, however, that convergence continues to hold if strict convexity is replaced with convexity. Only the assertion $d\left(m_{\delta}^{*}, M^{*}\right) \rightarrow 0$ has to be replaced by

$$
\sup _{x \in M_{\delta}^{*}} \inf _{y \in M^{*}}\|x-y\| \rightarrow 0
$$

where $M_{\delta}^{*}:=\operatorname{argmin}_{m \in \mathcal{C}} F_{\delta}(m)$. An analogous modification holds for Theorem 2 .

We close the section with the following result, which shows under additional regularity conditions on $F_{\delta}$ and $\mathcal{C}$ geometric, or, more precisely, at least $Q$-linear convergence of the GIRLS algorithm (cf. [3, Theorem 4.1], for a related result).

THEOREM 5 (geometric convergence of the GIRLS algorithm). Let $F_{\delta}: \mathbb{R}^{d} \rightarrow \mathbb{R}$ be coercive and twice continuously differentiable with positive definite Hessian matrix $D^{2} F\left(m_{\delta}^{*}\right)=:$. Further let $G_{\delta}: \mathbb{R}^{d} \times \mathbb{R}^{d}$ be a quadratic approximation of $F_{\delta}$ from above with Hessian matrix $B\left(m_{\delta}^{*}\right)=: B$ as in (13). Then the GIRLS algorithm yields a sequence $\left(m_{\delta}^{(k)}\right)_{k=0}^{\infty}$ converging to $m_{\delta}^{*}=\operatorname{argmin}_{\mathcal{C}} F_{\delta}$ such that

$$
\limsup _{k \rightarrow \infty} \frac{\left\|m_{\delta}^{(k+1)}-m_{\delta}^{*}\right\|_{A}}{\left\|m_{\delta}^{(k)}-m_{\delta}^{*}\right\|_{A}} \leq 1-\lambda_{\min }\left(B^{-1} A\right) \in[0,1) .
$$

Here $\|v\|_{A}:=\left(v^{\top} A v\right)^{1 / 2}$, and $\lambda_{\min }\left(B^{-1} A\right) \in(0,1]$ denotes the smallest eigenvalue of $B^{-1} A$. and

Proof. According to Theorem 2, $\lim _{k \rightarrow \infty} m_{\delta}^{(k)}=m_{\delta}^{*}$. Since $\mathcal{C}=\mathbb{R}^{d}, \nabla F_{\delta}\left(m_{\delta}^{*}\right)=0$

$$
\begin{aligned}
m_{\delta}^{(k+1)} & =m_{\delta}^{(k)}-B\left(m_{\delta}^{(k)}\right)^{-1} \nabla F_{\delta}\left(m_{\delta}^{(k)}\right) \\
& =m_{\delta}^{(k)}-B\left(m_{\delta}^{(k)}\right)^{-1} \int_{0}^{1} D^{2} F_{\delta}\left((1-t) m_{\delta}^{*}+t m_{\delta}^{(k)}\right)\left(m_{\delta}^{(k)}-m_{\delta}^{*}\right) \mathrm{d} t \\
& =m_{\delta}^{(k)}-B^{-1} A\left(m_{\delta}^{(k)}-m_{\delta}^{*}\right)+o\left(\left\|m_{\delta}^{(k)}-m_{\delta}^{*}\right\|\right) .
\end{aligned}
$$

Thus

$$
\frac{\left\|m_{\delta}^{(k+1)}-m_{\delta}^{*}\right\|_{A}}{\left\|m_{\delta}^{(k)}-m_{\delta}^{*}\right\|_{A}}=\frac{\left\|\left(I-B^{-1} A\right)\left(m_{\delta}^{(k)}-m_{\delta}^{*}\right)\right\|_{A}}{\left\|m_{\delta}^{(k)}-m_{\delta}^{*}\right\|_{A}}+o(1),
$$

and for any vector $v \in \mathbb{R}^{d}$,

$$
\begin{aligned}
\frac{\left\|\left(I-B^{-1} A\right) v\right\|_{A}^{2}}{\|v\|_{A}^{2}} & =\frac{v^{\top}\left(I-A B^{-1}\right) A\left(I-B^{-1} A\right) v}{v^{\top} A v} \\
& =\frac{w^{\top} A^{-1 / 2}\left(I-A B^{-1}\right) A\left(I-B^{-1} A\right) A^{-1 / 2} w}{\|w\|^{2}} \quad\left(\text { with } w:=A^{1 / 2} v\right) \\
& =\frac{w^{\top} C^{2} w}{\|w\|^{2}} \quad\left(\text { with } C:=I-A^{1 / 2} B^{-1} A^{1 / 2}\right) \\
& \leq \lambda_{\max }\left(C^{2}\right) .
\end{aligned}
$$

It follows from property (ii) of $G_{\delta}$ in Definition 2 that $B-A$ is nonnegative definite, which implies that $\lambda_{i}\left(B^{-1} A\right)=\lambda_{i}\left(A^{1 / 2} B^{-1} A^{1 / 2}\right) \in(0,1]$. This entails that $C$ is nonnegative definite with $\lambda_{\max }\left(C^{2}\right)=\lambda_{\max }(C)^{2}=\left(1-\lambda_{\min }\left(B^{-1} A\right)\right)^{2}$. 
TABLE 1

Generalized iteratively reweighted least squares algorithm (GIRLS).

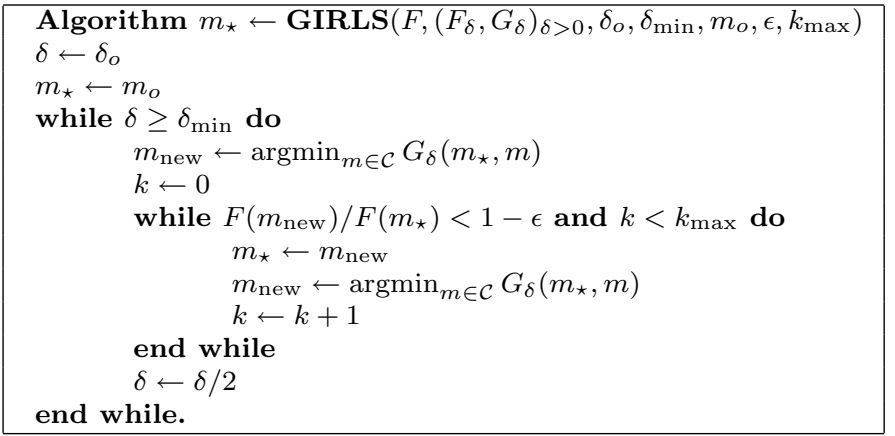

3.2. Pseudocode, proper choice of $\delta$, and the number of iterations. In practical applications the points $m_{\delta}^{*}$ are never calculated exactly. Instead after finitely many, say $k(\delta)$, iterations of (5) the iteration is terminated and the regularization parameter $\delta$ is decreased, e.g., replaced with $\delta / 2$. An obvious question is how to choose these iteration numbers $k(\delta)$. We found empirically in most cases that for a fixed parameter $\delta>0$, the values $F\left(m_{\delta}^{(k)}\right)$ are decreasing for $k \leq k_{o}(\delta)$ and increasing in $k \geq k_{o}(\delta)$ for some fixed $k_{o}(\delta) \in \mathbb{N}$. Hence in case of a strictly positive target function $F$ we may take

$$
k(\delta):=\min \left(\left\{k \in \mathbb{N}_{0}: F\left(m_{\delta}^{(k+1)}\right) / F\left(m_{\delta}^{(k)}\right) \geq 1-\epsilon\right\} \cup\left\{k_{\max }\right\}\right)
$$

for a small constant $\epsilon>0$ and a large maximal number $k_{\max }$. In the examples discussed subsequently, we found that for $\epsilon=10^{-5}$ and $k_{\max }=100$, the number $k(\delta)$ was never larger than 30 , which seems to compensate for the fact that the sequence $m_{\delta}^{(k)}$ converges only geometrically. This is similar to numerical findings with an implementation of an algorithm by [25, section 5] for the median and various parametric regression models.

Having determined $k(\delta)$ and $m_{\delta}^{(k(\delta))}$ for one particular $\delta>0$, we define $m_{\delta / 2}^{(0)}:=$ $m_{\delta}^{(k(\delta))}$ and repeat the same procedure with $\delta / 2$ in place of $\delta$, provided that $k(\delta)>0$. We proceed until $\delta / 2$ would be smaller than a certain threshold $\delta_{\min }$. Pseudocode for this algorithm is displayed in Table 1 . Input parameters are $F$, its regularization $\left(F_{\delta}, G_{\delta}\right)_{\delta>0}$ augmented with smooth aproximations from above, a starting value $\delta_{o}>0$ and a lower threshold $\delta_{\min } \in\left(0, \delta_{o}\right)$ for $\delta$, a starting point $m_{o} \in \mathcal{C}$, and a threshold $\epsilon>0$, as well as a maximal iteration number $k_{\max }$ for the inner while-loop.

4. Regularization and quadratic approximation for different types of regression problems. In the subsequent data examples the target functional $F(m)$ is always of type (1) or (3), i.e.,

$$
F(m)=\sum_{i=1}^{n} \rho\left(r_{i}(m)\right)+\lambda P(m)
$$

with $\lambda \geq 0$, where each residual $r_{i}(m)$ is an affine linear functional of $m \in \mathbb{R}^{d}$. Here each summand of $F$ is regularized and approximated separately. We will start with an auxiliary result justifying the quadratic approximation (9). 
Lemma 1. Let $h: \mathbb{R} \rightarrow \mathbb{R}$ be even and twice differentiable such that $h^{\prime \prime}$ is nonnegative and nonincreasing on $[0, \infty)$. For $r, z \in \mathbb{R}$ define

$$
g(r, z):=h(r)+\left(h^{\prime}(r) / r\right)\left(z^{2}-r^{2}\right) / 2,
$$

where $h^{\prime}(0) / 0:=h^{\prime \prime}(0)$. Then $g(r, z) \geq h(z)$ with equality if $z= \pm r$.

Proof. One verifies easily that $g(r, z)$ is even in both arguments with $g(r, r)=h(r)$. Thus it suffices to show that $g(r, z) \geq h(z)$ for any $r, z \geq 0$. Now,

$$
\begin{aligned}
g(r, z)-h(z) & =g(r, z)-h(r)-(h(z)-h(r)) \\
& =\left(h^{\prime}(r) / r\right)\left(z^{2}-r^{2}\right) / 2-h^{\prime}(r)(z-r)-\int_{r}^{z}\left(h^{\prime}(t)-h^{\prime}(r)\right) d t \\
& =\left(h^{\prime}(r) / r\right)(z-r)^{2} / 2-\int_{r}^{z}\left(h^{\prime}(t)-h^{\prime}(r)\right) d t \\
& =\int_{r}^{z}(\tilde{h}(r, 0)-\tilde{h}(r, t))(t-r) d t \\
& =\int_{\min (r, z)}^{\max (r, z)}(\tilde{h}(r, 0)-\tilde{h}(r, t))|t-r| d t,
\end{aligned}
$$

where $\tilde{h}(r, t):=\left(h^{\prime}(t)-h^{\prime}(r)\right) /(t-r)$ for $t \neq r$, and $\tilde{h}(r, r):=h^{\prime \prime}(r)$. One can deduce easily from $h^{\prime \prime}$ being nonincreasing on $[0, \infty)$ that $\tilde{h}(r, \cdot)$ has the same property. Thus the integrand of (16) is nonnegative.

Let us first describe how to approximate $\rho$ itself in three special cases. After this we will discuss several penalizations $P$ in (15). Finally we comment on isotonic regression, an example with $\mathcal{C} \neq \mathbb{R}^{d}$.

Quantile regression. Let $\rho(z)$ be given by (2). This may be rewritten as

$$
\rho(z)=|z|+(2 p-1) z .
$$

Hence we utilize the functions $h_{\delta}$ and $g_{\delta}$ from (7) and (9), which yields the regularization

$$
z \mapsto h_{\delta}(z)+(2 p-1) z,
$$

and by means of Lemma 1 the quadratic approximation

$$
z \mapsto g_{\delta}(r, z)+(2 p-1) z=c_{\delta}(r)+h_{\delta}(r)^{-1} z^{2} / 2+(2 p-1) z
$$

of $z \mapsto \rho(z)$, where $c_{\delta}(r)$ is an irrelevant constant.

$\boldsymbol{L}^{\boldsymbol{q}}$-regression. Let $\rho(z):=|z|^{q}$ for some $q \in[1, \infty)$. If $1 \leq q<2$, one may generalize definitions (7) and (9) immediately as follows:

$$
\begin{aligned}
h_{\delta}(z) & :=\left(z^{2}+\delta\right)^{q / 2} \\
g_{\delta}(r, z) & :=h_{\delta}(r)+q\left(r^{2}+\delta\right)^{1-q}\left(z^{2}-r^{2}\right) / 2 \\
& =c_{\delta}(r)+q\left(r^{2}+\delta\right)^{1-q} z^{2} / 2 .
\end{aligned}
$$

Again it follows from Lemma 1 that $g_{\delta}(r, z) \geq h_{\delta}(z)$ with equality for $z= \pm r$.

In case of $q>2$, the second derivative of $z \mapsto|z|^{q}$ is increasing in $|z|$ and unbounded, and hence Lemma 1 cannot be applied directly. To circumvent this problem, one could redefine

$$
h_{\delta}(z):=\left\{\begin{array}{cl}
|z|^{q} & \text { if }|z| \leq \delta^{-1} \\
a_{\delta}+b_{\delta}|z|+q(q-1) \delta^{2-q} z^{2} / 2 & \text { otherwise }
\end{array}\right.
$$

Copyright $@$ by SIAM. Unauthorized reproduction of this article is prohibited. 
with constants $a_{\delta}, b_{\delta}$ such that $h_{\delta}$ is twice continuously differentiable, and then use the quadratic approximation

$$
g_{\delta}(r, z):=h_{\delta}(r)+h_{\delta}^{\prime}(r)(z-r)+q(q-1) \delta^{2-q}(z-r)^{2} / 2 .
$$

Logistic regression. For data sets with a covariable $X$ and a dichotomous response $Y \in\{0,1\}$, maximum likelihood estimation of $M(X):=\log [P(Y=1 \mid X) /$ $P(Y=0 \mid X)]$ involves "residuals" $z=(1 / 2-Y) M(X)$ and

$$
\rho(z):=h(z)+z \quad \text { with } \quad h(z):=\log \left[e^{z}+e^{-z}\right] .
$$

Note that $h$ satisfies the conditions of Lemma 1 with $h^{\prime}(r)=\tanh (r)$ and $h^{\prime \prime}(r)=$ $1-\tanh (r)^{2}$. Thus regularization is superfluous, while quadratic approximation is straightforward. In this case, the well-known IRLS algorithm results ([28]).

Roughness penalties. Let us start with two particular examples for $P(m)$. For given real numbers $x_{1}<x_{2}<\cdots<x_{d}$ let $M$ be a function on $\left[x_{1}, x_{d}\right]$ and $m:=\left(M\left(x_{j}\right)\right)_{j=1}^{d}$. Then let

$$
\begin{aligned}
\mathrm{TV}^{(0)}(m) & :=\sum_{j=1}^{d-1}\left|m_{j}-m_{j+1}\right| \\
\mathrm{TV}^{(1)}(m) & :=\sum_{j=2}^{d-1}\left|\Delta_{j} m\right| \quad \text { with } \quad \Delta_{j} m:=\frac{m_{j+1}-m_{j}}{x_{j+1}-x_{j}}-\frac{m_{j}-m_{j-1}}{x_{j}-x_{j-1}} .
\end{aligned}
$$

If $M$ is continuous and piecewise linear with knots in $\left\{x_{1}, \ldots, x_{d}\right\}$, then $\operatorname{TV}^{(0)}(m)$ and $\mathrm{TV}^{(1)}(m)$ are the total variation of $M$ and its first derivative, respectively. One could also think about smoother functions $M$ and approximate the total variation of its second or higher order derivative by suitable divided differences of $m$.

Generally, let $P(m)$ be a sum of several functionals of the form

$$
m \mapsto\left|v^{\top} m\right|
$$

with a given vector $v \in \mathbb{R}^{d} \backslash\{0\}$. For instance, $\mathrm{TV}^{(0)}(m)$ involves

$$
v_{i}=v_{i}^{(j)}:=\left\{\begin{array}{cl}
1 & \text { if } i=j \\
-1 & \text { if } i=j+1 \\
0 & \text { else }
\end{array}\right.
$$

for $1 \leq j<d$, while $\operatorname{TV}^{(1)}(m)$ involves

$$
v_{i}=v_{i}^{(j)}:=\left\{\begin{array}{cl}
\left(x_{j}-x_{j-1}\right)^{-1} & \text { if } i=j-1, \\
-\left(x_{j}-x_{j-1}\right)^{-1}-\left(x_{j+1}-x_{j}\right)^{-1} & \text { if } i=j, \\
\left(x_{j+1}-x_{j}\right)^{-1} & \text { if } i=j+1, \\
0 & \text { else }
\end{array}\right.
$$

for $1<j<d$. Now an obvious strategy is to regularize $m \mapsto\left|v^{\top} m\right|$ by $m \mapsto h_{\delta}\left(v^{\top} m\right)$ and approximate this quadratically by

$$
m \mapsto g_{\delta}\left(v^{\top} f, v^{\top} m\right)=c_{\delta}\left(v^{\top} f\right)+h_{\delta}\left(v^{\top} f\right)^{-1}\left(v^{\top} m\right)^{2} / 2 .
$$

Copyright (c) by SIAM. Unauthorized reproduction of this article is prohibited. 
Often it is desirable to work with quadratic approximations $G(f, \cdot)$ whose Hessian matrix $B(f)$ is diagonal. For that purpose one can modify the quadratic term $Q(m):=$ $\left(v^{\top} m\right)^{2}$ as follows:

$$
\begin{aligned}
Q(m) & =\left(v^{\top} f\right)^{2}+2 f^{\top} v v^{\top}(m-f)+\left(v^{\top}(m-f)\right)^{2} \\
& \leq\left(v^{\top} f\right)^{2}+2 f^{\top} v v^{\top}(m-f)+\|v\|^{2} \sum_{i: v_{i} \neq 0}\left(m_{i}-f_{i}\right)^{2} \\
& =c(v, f)-2 w(v, f)^{\top} m+\|v\|^{2} \sum_{i: v_{i} \neq 0} m_{i}^{2}
\end{aligned}
$$

for some irrelevant constant $c(v, f)$ and $w(v, f)_{i}:=1_{v_{i} \neq 0}\|v\|^{2} f_{i}-v^{\top} f v_{i}$.

Isotonic regression. In some applications one seeks to minimize a functional such as (15) over all vectors in $\mathcal{C} \nearrow:=\left\{m \in \mathbb{R}^{d}: m_{1} \leq \cdots \leq m_{d}\right\}$. In the simplest case, $d=n$ and $\rho\left(r_{i}(m)\right)=\left(Y_{i}-m_{i}\right)^{q}$ for some $q \in[1, \infty]$, where $q=\infty$ corresponds to supremum norm of $Y-m$. For this special case it is well known (see [2]) that an explicit solution exists only for $q=1,2, \infty$. In general, via regularization and suitable quadratic approximation from above, each updating step of the GIRLS algorithm involves minimization of

$$
G_{\delta}(f, m)=C(f)+\sum_{i=1}^{d} w_{i}(f)\left(m_{i}-b_{i}(f)\right)^{2}
$$

over all vectors $m \in \mathcal{C}$, with certain weights $w_{i}(f)>0$, an irrelevant constant $C(f)$, and certain numbers $b_{i}(f)$. This minimization problem can be solved explicitly by means of the PAVA ([33]).

5. Numerical examples. In this section we discuss the numerical performance of the GIRLS algorithm in practical applications. Our first example is about quantile regression and shows that GIRLS outperforms a Newton-Raphson type algorithm, at least in the first iterations. In the second example we consider a unimodal regression problem. For ordinary isotonic regression in one-dimensional settings, PAVA is known to be highly efficient (cf., e.g., [33]) in the sense of producing an exact solution in $O(d)$ steps. Replacing the isotonicity constraint on $m$ with a penalty term $\operatorname{TV}^{(0)}(m)$ leads to minimization problems which can be solved efficiently with the closely related "taut string algorithm" (cf. [8]). However, analogous problems in multidimensional regression settings, or using $\mathrm{TV}^{(k)}(m)$ with $k \geq 1$, are computationally more involved. We mention, e.g., [15] for a reformulation as a bilateral contact problem and a rather involved solution by a two-level iterative method requiring a semi-smooth Newton method and a primal dual active set algorithm. GIRLS offers a different strategy: PAVA can be applied after approximating the target functional suitably by a quadratic program; hence, we can also understand GIRLS as a linking device to apply efficient special purpose algorithms for quadratic programs such as PAVA to corresponding nonquadratic and/or constrained problems.

Example 1 (estimation of smooth quantile functions). We applied the GIRLS algorithm and a competitor to a dataset containing the income $(X)$ and the expenditure for food $(Y)$ in the year 1973 for 7125 households in Great Britain (Family Expenditure Survey 1968-1983). This dataset has also been analyzed by [14]. In order to enhance the visual quality, we reduced these data to a random subset of size $n=2000$. Moreover, since the empirical distributions of both variables are 


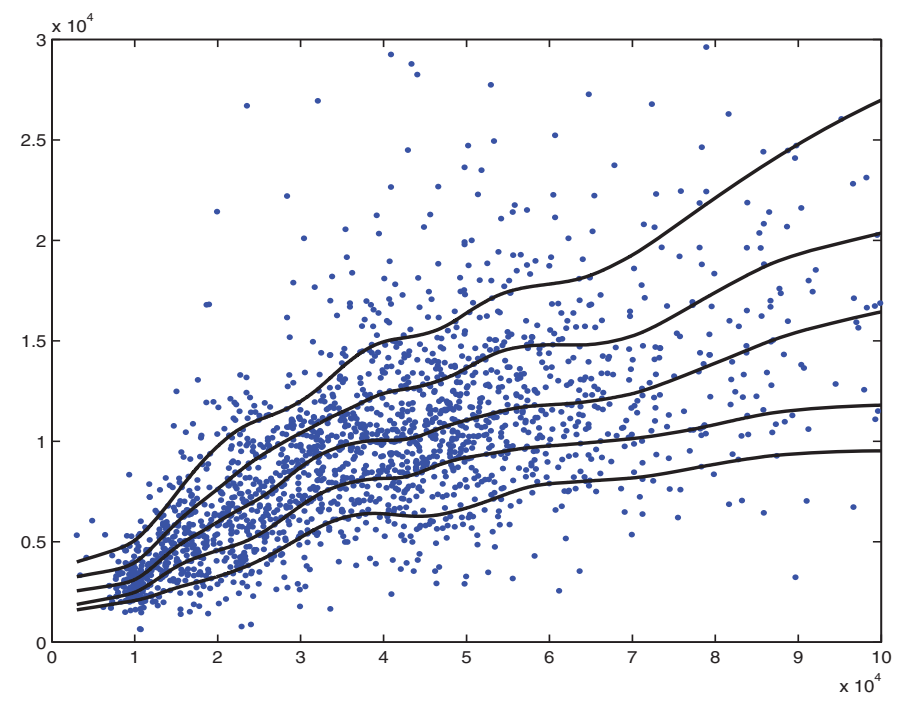

FIG. 1. Quantile curves for food expenditure data.

strongly skewed to the right, we plotted only the 1936 pairs $\left(X_{i}, Y_{i}\right)$ in the range $\left[0,10^{5}\right] \times\left[0,3 \cdot 10^{4}\right]$. Figure 1 shows the data together with estimated $p$-quantile curves $M_{p}$ for $p=0.1,0.25,0.5,0.75,0.9$. Here we used the functional

$$
F(m)=\sum_{i=1}^{n} \rho\left(Y_{i}-m_{C(i)}\right)+\lambda \mathrm{TV}^{(1)}(m)
$$

on $\mathbb{R}^{d}$ with $\rho$ given by $(2)$, where $x_{1}<x_{2}<\cdots<x_{d}$ are the $d=1945$ different $X_{-}^{-}$ values in the sample, $m_{j}=M_{p}\left(x_{j}\right), X_{i}=x_{C(i)}$, and $F$ was regularized as discussed in the last section. The tuning parameter $\lambda$ was chosen to be $2 \cdot 10^{6}$ by visual inspection.

We now turn to the numerical performance of the GIRLS algorithm and compare it to a Newton-Raphson algorithm (robustified with a standard step-size correction as in [12]). In the first step we compare the performance of the two algorithms in the particular setting of our data example; the second step will consist of a small simulation study with artificial data. All computations were performed with Matlab on a $1.86 \mathrm{Ghz}$ Pentium-processor with 1GB Ram. As starting value of the iterations we used the polynomial regression $p$-quantile of order 1 , and the tuning parameter $\delta$ was selected in a data-driven way from this starting value as the smaller of the median of its absolute residuals, and the median of its first order differences, in both cases divided by 1000. In the first step, we compared the computational efficiency of the GIRLS and the Newton-Raphson algorithm applied to quantile regression with the family expenditure data. To this end we recorded the computing times and number of iterations required for determination of the 25\%-quantile curve by GIRLS and the Newton-Raphson algorithm, where we stopped the iterations as soon as the relative improvement of the function $F(m)$ between two subsequent iterations fell below a threshold parameter $\varepsilon=10^{-12}$. Whereas GIRLS required 5.0s CPU time and 59 iterations to find the solution, the Newton-Raphson algorithm turned out to be significantly more expensive with $46.4 s \mathrm{CPU}$ time and 425 iterations. We also performed the same computations for a wide range of threshold parameters $\varepsilon=$ $10^{-6} \ldots 10^{-24}$, without significant change in the relative computational expense of the two methods. 


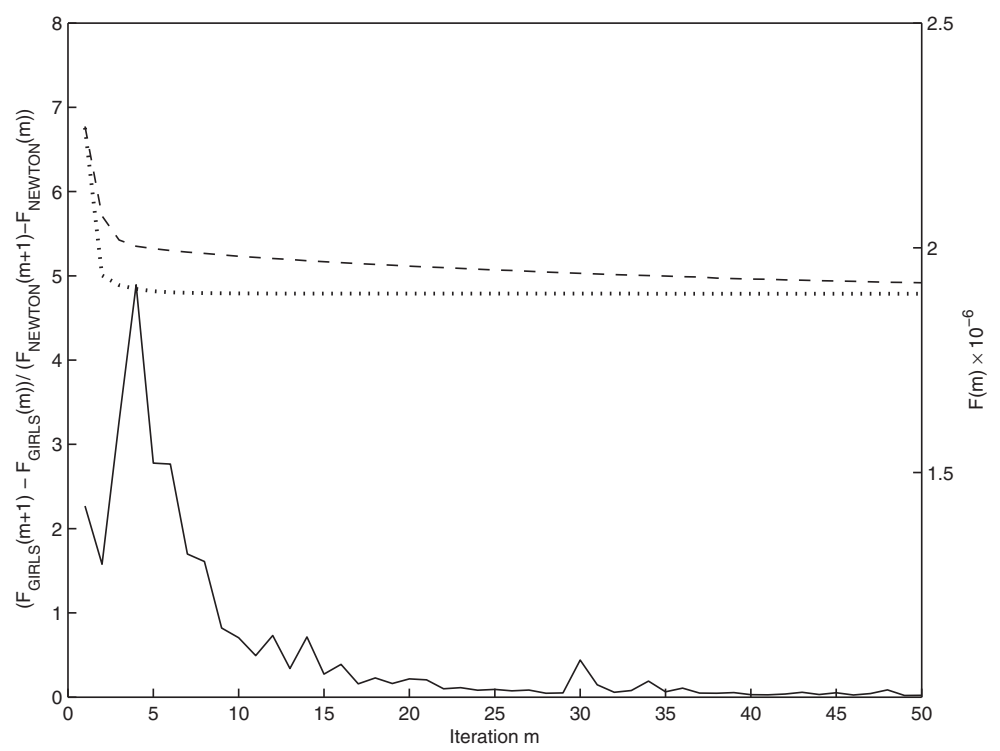

FIG. 2. Mean performance of GIRLS and a Newton-Raphson-algorithm. The dashed and dotted curves show the mean value of the function $F(m)$ in dependence of the iteration number $m$ for the Newton-Raphson algorithm and GIRLS, respectively, and the solid curve the stepwise rate of improvement $\left(F_{\mathrm{GIRLS}}(m+1)-F_{\mathrm{GIRLS}}(m)\right) /\left(F_{\mathrm{NEWTON}}(m+1)-F_{\mathrm{NEWTON}}(m)\right)$ of $F(m)$ by the two methods. Here, a value larger than 1 indicates that in the $m$ th iteration $F(m)$ decreased more for GIRLS than for the Newton-Raphson-algorithm.

In the second step of our analysis we performed 100 simulations of the quantile regression problem with (artificial) regression data from the model

$$
Y_{i}=\sin \left(X_{i} \cdot \frac{\pi}{2}\right)+\varepsilon_{i}, i=1, \ldots, 1000 .
$$

Here, $X_{i} \sim U[0,1]$ are uniformly distributed, independent design variables, and $\varepsilon_{i} \sim$ $N(0,0.01)$ i.i.d. noise terms. We used both GIRLS and the Newton-Raphson method to estimate the $25 \%$-quantile curve of the data by determining the minimizer of the function $F(m)$. From visual inspection of a pilot simulation, we chose the tuning parameter $\lambda=2 \cdot 10^{4}$ for the subsequent simulations.

Figure 2 compares the mean performance of the GIRLS algorithm with the performance of the Newton-Raphson-method with step-size correction. The two curves in the top show the value of the function $F(m)$ in dependence of the iteration number $m$, and the bottom curve represents the mean improvement (in the simulations) of the solution, measured by the decrease of the target function $F(m)$. From the figure, we conclude that the first steps of GIRLS are significantly more efficient than for the Newton-Raphson method, whereas the latter catches up after approximately the $8^{\text {th }}$ iteration.

In summary, in the computations with the penalized quantile regression problem, GIRLS outperformed the Newton-Raphson method for the family expenditure data. Moreover, in our simulation we found GIRLS to improve the solution much faster than the Newton-Raphson method in the first $\approx 8$ iterations. Only if the initial value of the Newton algorithm has been chosen very close to the true minimizer $M^{*}$, we found the performance of the Newton algorithm to be superior. Hence, for practical purposes it seems be advisable to combine both algorithms such that GIRLS will be 
used at least as an initial algorithm which efficiently provides a good initial value for a subsequently performed Newton-type algorithm. Moreover, if both GIRLS and the Newton-Raphson method are available, it may be useful to compute both a GIRLS and a Newton step, with subsequent selection of the better one.

Example 2 (GIRLS as a device to utilize efficient algorithms for special quadratic programs in more complex settings). In our second example we will briefly illustrate the flexibility of the GIRLS algorithm to combine several constraints. Precisely, we combine unimodality constraints with TV penalization. Ordinary isotonic regression and hence unimodal regression involves the solution of a weighted least squares problem, and efficient algorithms, the PAVA in particular, are available. If we add a TV penalty, the problem is no longer a quadratic program, and PAVA is not applicable directly. By replacing the problem to be solved by a sequence of quadratic programs, GIRLS makes it possible again to apply PAVA for unimodal regression with TV penalization. To this end consider a two-dimensional regression problem where $Y_{i j}$ are observations to be fitted by $m=\left(m_{i j}\right)_{i, j}, i=1, \ldots, m, j=1, \ldots, n$. We want to minimize the sum $F(m)$ of the quadratic cost functional

$$
\|Y-m\|^{2}=\sum_{i j}\left(Y_{i j}-m_{i j}\right)^{2}
$$

and the total variation penalty $\lambda \operatorname{TV}(m)$, where $\lambda>0$ and

$$
\mathrm{TV}(m):=\sum_{i=1}^{m} \sum_{j=1}^{n}\left|m_{i+1, j}-m_{i j}\right|+\sum_{i=1}^{m} \sum_{j=1}^{n}\left|m_{i, j+1}-m_{i j}\right| .
$$

For the regularization $F_{\delta}$ and quadratic approximation $G_{\delta}(f, \cdot)$, the least squares term is kept unchanged, while each summand $\left|m_{(a)}-m_{(b)}\right|$ of $\operatorname{TV}(m)$ is treated as described in section 4: First we regularize it by $h_{\delta}\left(m_{(a)}-m_{(b)}\right)$. Then as a first quadratic approximation we may use

$$
g_{\delta}\left(f_{(a)}-f_{(b)}, m_{(a)}-m_{(b)}\right)=C_{\delta,(a),(b)}(f)+\frac{\left(m_{(a)}-m_{(b)}\right)^{2}}{2 h_{\delta}\left(f_{(a)}-f_{(b)}\right)} .
$$

For our purposes it turns out to be more suitable to replace the enumerator $\left(m_{(a)}-\right.$ $\left.m_{(b)}\right)^{2}$ with

$$
2\left(m_{(a)}-\frac{f_{(a)}+f_{(b)}}{2}\right)^{2}+2\left(m_{(b)}-\frac{f_{(a)}+f_{(b)}}{2}\right)^{2},
$$

which is never less than $\left(m_{(a)}-m_{(b)}\right)^{2}$ with equality for $m=f$. The advantages of this latter quadratic approximation are computational simplicity and feasibility of isotonic (and hence unimodal) least squares algorithms. This allows us, e.g., to impose in addition unimodality in vertical direction. Hence, simply in each step for each vertical line the unimodal regression is calculated by means of some standard variation of the PAVA.

Acknowledgments. The authors are thankful to the associate editor and three anonymous referees for their constructive criticism that helped to improve this paper significantly. Moreover, they would like to thank G. Jongbloed and O. Scherzer for helpful comments. The third author is indebted to Y. Vardi for interesting discussions on a previous version of this paper. Parts of this paper were written while 
L. Dümbgen was visiting Göttingen University as lecturer within the PhD Program "Applied Statistics and Empirical Methods," and while N. Bissantz was visiting the University of Bern.

\section{REFERENCES}

[1] R. E. Barlow, D. J. Bartholomew, J. M. Bremner, and H. D. Brunk, Statistical Inference Under Order Restrictions. The Theory and Application of Isotonic Regression, John Wiley \& Sons, London, New York, Sydney, 1972.

[2] R. E. Barlow and V. Ubhaya, Isotonic approximation, in Optimisation Methods in Statistics, J. S. Rustagi, ed., Academic Press, 1971, New York, pp. 77-86.

[3] D. Böhning And B. Lindsay, Monotonicity of quadratic-approximation algorithms, Ann. Inst. Statist. Math., 40 (1988), pp. 641-663.

[4] A. W. Bowman and A. Azzalini, Applied Smoothing Techniques for Data Analysis: The Kernel Approach with S-Plus Illustrations, Oxford University Press, Oxford, UK, 1997.

[5] B. M. Brown, Statistical uses of the spatial median, J. Roy. Statist. Soc. Ser. B, 45 (1983), pp. 25-30.

[6] B. M. Brown, P. Hall, And G. A. Young, On the effect of inliers on the spatial median, J. Multivariate Anal., 63 (1997), pp. 88-104.

[7] D. Coleman, P. Holland, N. Kaden, V. Klema, and S. C. Peters, A system of subroutines for iteratively reweighted least squares computations, ACM Trans. Math. Software, 6 (1980), pp. 327-336.

[8] P. L. Davies AND A. Kovac, Local extremes, runs, strings and multiresolution (With discussion and rejoinder by the authors), Ann. Statist., 29 (2001), pp. 1-65.

[9] J. DE Leeuw And G. Michailidis, Discussion article on the paper by Lange, Hunter \& Y Yang, J. Comput. Graph. Statist., 9 (2000), pp. 26-31.

[10] Y. Dodge And J. JurečKovÁ, Adaptive Regression, Springer-Verlag, New York, 2000.

[11] G. R. Ducharme and P. Milasevic, Spatial median and directional data, Biometrika, 74 (1987), pp. 212-215.

[12] L. Dümbgen, S. Freitag-Wolf, and G. Jongbloed, Estimating a unimodal distribution from interval-censored data, J. Amer. Statist. Assoc., 101 (2006), pp. 1094-1106.

[13] U. EcKHARDT, Weber's problem and Weiszfeld's algorithm in general spaces, Math. Program., 18 (1980), pp. 186-196.

[14] W. HÄrdle AND J. S. MARron, Bootstrap simultaneous error bars for nonparametric regression, Ann. Statist., 19 (1991), pp. 778-796.

[15] W. Hinterberger, M. Hintermüller, K. Kunisch, M. von Oehsen, and O. Scherzer, Tube methods for $B V$ regularisation, J. Math. Imaging Vision, 19 (2003), pp. 219-235.

[16] P. J. Huber, Robust estimation of a location parameter, Ann. Math. Stat., 35 (1964), pp. 73101.

[17] P. J. Huber, Robust Statistics, John Wiley \& Sons Inc., New York, 1981.

[18] D. R. Hunter And K. LAnge, Quantile Regression via an MM Algorithm, J. Comput. Graph. Statist., 9 (2000), pp. 60-77.

[19] I. N. Katz, Local convergence in Fermat's problem, Math. Program., 6 (1974), pp. 89-104.

[20] K. Lange, D. R. Hunter, And I. Yang, Optimization Transfer Using Surrogate Objective Functions, J. Comput. Graph. Statist., 9 (2000), pp. 1-20.

[21] R. Koenker and G. Bassett, Regression quantiles, Econometrica, 46 (1978), pp. 33-50.

[22] R. Koenker, P. NG, And S. Portnoy, Quantile smoothing splines, Biometrika, 81 (1994), pp. $673-680$.

[23] H. R. KüNSCH, Robust priors for smoothing and image restoration, Ann. Inst. Statist. Math., 46 (1994), pp. 1-19.

[24] H. W. Kunn, A note on Fermat's problem, Math. Program., 4 (1973), pp. 98-107.

[25] M. G. Lejeune And P. SARDA, Quantile regression: A nonparametric approach, Comput. Statist. Data Anal., 6 (1988), pp. 229-239.

[26] E. Mammen, J. S. Marron, B. A. Turlach, and M. P. Wand, A general projection framework for constrained smoothing, Statist. Sci., 16 (2001), pp. 232-248.

[27] E. Mammen and S. van de Geer, Locally adaptive regression splines, Ann. Statist., 25 (1997), pp. $387-413$.

[28] P. McCullagh and J. Nelder, Generalized Linear Models, 2nd ed., Chapman \& Hall, London, 1989.

[29] D. P. O'LEARY, Robust regression computation using iteratively reweighted least squares, SIAM J. Matrix Anal. Appl., 11 (1990), pp. 466-480.

Copyright (c) by SIAM. Unauthorized reproduction of this article is prohibited. 
[30] S. Portnoy, Local asymptotics for quantile smoothing splines, Ann. Statist., 25 (1997), pp. 414-434.

[31] D. A. Ratkowsky, Nonlinear Regression Modelling, Dekker, New York, 1983.

[32] T. Robertson and P. Waltman, On estimating monotone parameters, Ann. Math. Statist., 39 (1968), pp. 1030-1039.

[33] T. Robertson, F. T. Wright, and R. L. Dykstra, Order Restricted Statistical Inference, John Wiley \& Sons Ltd., Chichester, UK, 1988.

[34] R. T. Rockafellar, Convex Analysis, Princeton University Press, Princeton, NJ, 1970.

[35] E. Stein and R. Shakarchi, Real Analysis, Princeton Lectures in Analysis, 2005.

[36] Y. VARdi And C.-H. Zhang, The multivariate $L_{1}$-median and associated data depth, Proc. Natl. Acad. Sci. USA, 97 (2000), pp. 1423-1426.

[37] Y. VARdi And C-H. Zhang, A modified Weiszfeld algorithm for the Fermat-Weber location problem, Math. Program. Ser. A, 90 (2001), pp. 559-566.

[38] C. R. Vogel And M. E. Oman, Iterative methods for total variation denoising, SIAM J. Sci. Comput., 17 (1996), pp. 227-238.

[39] H. Voss AND U. ECKHARDT, Linear Convergence of generalized Weiszfeld's method, Computing, 25 (1980), pp. 243-251.

[40] E. Weiszfeld, Sur un problème de minimum dans l'espace, Tohoku Math. J., 42 (1936), pp. $274-280$.

[41] E. Weiszfeld, Sur le point pour lequel la somme des distances de $n$ points donnés est minimum, Tohoku Math. J., 43 (1937), pp. 355-386.

[42] R. Wolke And H. Schwetlick, Iteratively reweighted least squares: Algorithms, convergence analysis, and numerical comparisons, SIAM J. Sci. Statist. Comput., 9 (1988), pp. 907921.

Copyright (c) by SIAM. Unauthorized reproduction of this article is prohibited. 